\title{
Quantum to classical transition in a
}

\section{single-ion laser}

\author{
François Dubin ${ }^{1,2}$, Carlos Russo ${ }^{1 \dagger}$, Helena G. Barros ${ }^{1,3}$, Andreas Stute ${ }^{1,3}$, Christoph Becher ${ }^{1 \dagger}$, \\ Piet O. Schmidt ${ }^{1 \star \dagger}$ and Rainer Blatt ${ }^{1,3}$
}

\begin{abstract}
The onset of lasing in a 'classical' laser' is marked by a threshold that can be characterized by a sharp increase in photon flux as a function of external pumping strength ${ }^{2,3}$. The same is not necessarily true for a single trapped atom interacting with a single optical mode ${ }^{4-6}$. 'Quantum' lasers have shown thresholdless lasing in the regime of strong coupling between an atom and a radiation field 7,8 , but the existence of a threshold has been predicted ${ }^{9-12}$. Here, we demonstrate and characterize a single-atom laser with and without threshold behaviour by changing the strength of atom/light-field coupling. We observe the establishment of a laser threshold through the accumulation of photons in the optical mode even for a mean photon number substantially lower than for the classical case. Our observations mark an important step towards a fundamental understanding of laser operation in the few-atom limit ${ }^{13}$ including systems based on semiconductor quantum dots ${ }^{14}$ or molecules ${ }^{15}$.
\end{abstract}

Conventional lasers reach their threshold when the rate at which photons are coherently fed into the cavity mode exceeds losses and stimulated emission dominates over spontaneous emission into the lasing mode $e^{2,3}$. The most prominent characteristic of this threshold is a steep increase of photon flux from the laser while increasing the strength of the external pump exciting the gain medium. At the same time, intensity fluctuations are reduced ${ }^{11,12,16,17}$. These characteristics are induced by the nonlinear process of photonstimulated emission of photons into the lasing mode. At the singleatom level, characteristics that strongly deviate from standard laser properties have been predicted ${ }^{11,12,16,18,19}$, in particular regarding the question of the existence of a threshold. For strong atom-cavity coupling, no threshold exists. In this regime, photons are coherently scattered into the cavity mode through stimulation by the vacuum field ${ }^{8}$. More importantly, strong coupling introduces a photon blockade, which suppresses cavity-mode populations with more than one photon ${ }^{20}$. As a consequence, photon-stimulated emission enabling the threshold is absent, the laser threshold disappears and non-classical radiation is emitted ${ }^{8,9,12,21}$. It was pointed out theoretically that a one-atom laser can show a threshold ${ }^{11,12}$ in an intermediate-coupling regime. Here we explore a single-ion device in this regime that shows a laser threshold for strong external pumping for which photon-stimulated processes become important, owing to the non-zero population of cavity modes with more than one photon.

The schematic experimental set-up of our ion-trap cavity quantum electrodynamics system is shown in Fig. 1a. Using frequency- and intensity-stabilized lasers, we excite a single ${ }^{40} \mathrm{Ca}^{+}$ ion that is confined in a linear Paul trap at the centre of a near-concentric optical cavity ${ }^{22}$. The cavity provides a coherent coupling rate between the ion and a selected optical mode of $g / 2 \pi=1.3(1) \mathrm{MHz}$, limited by the localization of the ion along the cavity standing wave. With a cavity finesse of $\approx 70,000$ at $866 \mathrm{~nm}$, the field decay rate from the cavity is given by $\kappa / 2 \pi=54(1) \mathrm{kHz}$. A Hanbury-Brown and Twiss set-up consisting of a beamsplitter and two avalanche photodiodes is used to analyse photons leaving the cavity. It provides us with the steady-state population of photons in the cavity ${ }^{22}, n_{\mathrm{ss}}$, and allows us to compute the second-order correlation function, $g^{(2)}(\tau)$ (see Supplementary Information).

Figure $1 \mathrm{~b}$ shows a reduced three-level scheme of the ${ }^{40} \mathrm{Ca}^{+}$ion's electronic structure, which allows us to qualitatively describe the excitation process. Using an off-resonant drive laser, we achieve an effective coupling on a vacuum-stimulated Raman transition ${ }^{4}$ between the electronic ground state $S_{1 / 2}$ and the metastable state $D_{3 / 2}$. A transfer from $S_{1 / 2}$ to $D_{3 / 2}$ deposits a photon in the cavity. The effective coupling between these levels is $g_{\text {eff }} \sim g \cdot s_{1}$, where we have defined the drive parameter $s_{1} \equiv \Omega_{1} / 2\left|\Delta_{1}\right|$, with $\Omega_{1}$ denoting the Rabi frequency of the $397 \mathrm{~nm}$ driving laser and $\Delta_{1}$ its fixed detuning from the $S_{1 / 2}-P_{1 / 2}$ resonance. To close the excitation cycle, an infrared recycling laser at $866 \mathrm{~nm}$, with a resonant Rabi frequency $\Omega_{2}$ and detuning $\Delta_{2}$, transfers population from the $D_{3 / 2}$ to the $P_{1 / 2}$ state. From there, spontaneous emission projects the ion back to the $S_{1 / 2}$ ground state at a rate $\left(2 \gamma_{1} / 2 \pi\right)=20 \mathrm{MHz}$. The corresponding recycling rate is given by $2 \gamma_{\mathrm{r}}=2 \gamma_{1} s_{2}^{2}$ with the recycling parameter $s_{2} \equiv \Omega_{2} / 2 \widetilde{\Delta}_{2}$. Here, we define the effective detuning $\Delta_{2} \equiv \sqrt{\Delta_{2}^{2}+\left(\Omega_{2} / 2\right)^{2}+\gamma^{2}}$, where $2 \gamma=2\left(\gamma_{1}+\gamma_{2}\right)$ is the total decay rate from the $P_{1 / 2}$ state and $2 \gamma_{2} / 2 \pi=1.69 \mathrm{MHz}$ is the decay rate back to the $D_{3 / 2}$ state. The total cycling rate $\Gamma_{\text {tot }}$ for external pumping, that is, the rate at which one photon is added to the cavity mode, is thus given by $1 / \Gamma_{\text {tot }} \sim 1 / 2 g_{\text {eff }}+1 / 2 \gamma_{\mathrm{r}}$. The strength of external pumping is then controlled by the drive and recycling lasers; however, note that the drive parameter $s_{1}$ is kept constant in our experiments where the external pumping strength is varied only by the recycling parameter. Furthermore, the drive and recycling lasers introduce the broadenings $\gamma_{\text {eff }}^{(1)} \sim \gamma s_{1}^{2}$ and $\gamma_{\text {eff }}^{(2)} \sim \gamma s_{2}^{2}$, of the $S_{1 / 2}$ and $D_{3 / 2}$ states, respectively. The total linewidth of the $S_{1 / 2} \leftrightarrow D_{3 / 2}$ Raman transition is therefore given by $2 \gamma_{\text {eff }}=2\left(\gamma_{\text {eff }}^{(1)}+\gamma_{\text {eff }}^{(2)}\right)=2 \gamma\left(s_{1}^{2}+s_{2}^{2}\right)$. In this situation, an effective strong coupling is achieved for $g_{\text {eff }}>\left(\gamma_{\text {eff }}, \kappa\right)$. In the following experiments, this condition is fulfilled for weak external pumping at a fixed detuning of the drive laser $\left(\Delta_{1} / 2 \pi \approx 350 \mathrm{MHz}\right)$. Note that

\footnotetext{
${ }^{1}$ Institut für Experimentalphysik, Universität Innsbruck, Technikerstr. 25, 6020 Innsbruck, Austria, ${ }^{2}$ ICFO-Institut de Ciències Fotòniques, Mediterranean Technology Park, 08860 Castelldefels, Spain, ${ }^{3}$ Institut für Quantenoptik und Quanteninformation, Österreichische Akademie der Wissenschaften, Otto-Hittmair-Platz 1, 6020 Innsbruck, Austria. †Present addresses: GoLP/Instituto de Plasmas e Fusão Nuclear, Instituto Superior Técnico, Av., Rovisco Pais, 1049-001 Lisboa, Portugal (C.R.); Fachrichtung Technische Physik, Universität des Saarlandes, Campus E2.6, 66123 Saarbrücken, Germany (C.B.); QUEST Institut für Experimentelle Quantenmetrologie, Physikalisch-Technische Bundesanstalt und Leibniz Universität Hannover, Bundesallee 100,38116 Braunschweig, Germany (P.O.S.). *e-mail: Piet.Schmidt@ptb.de.
} 

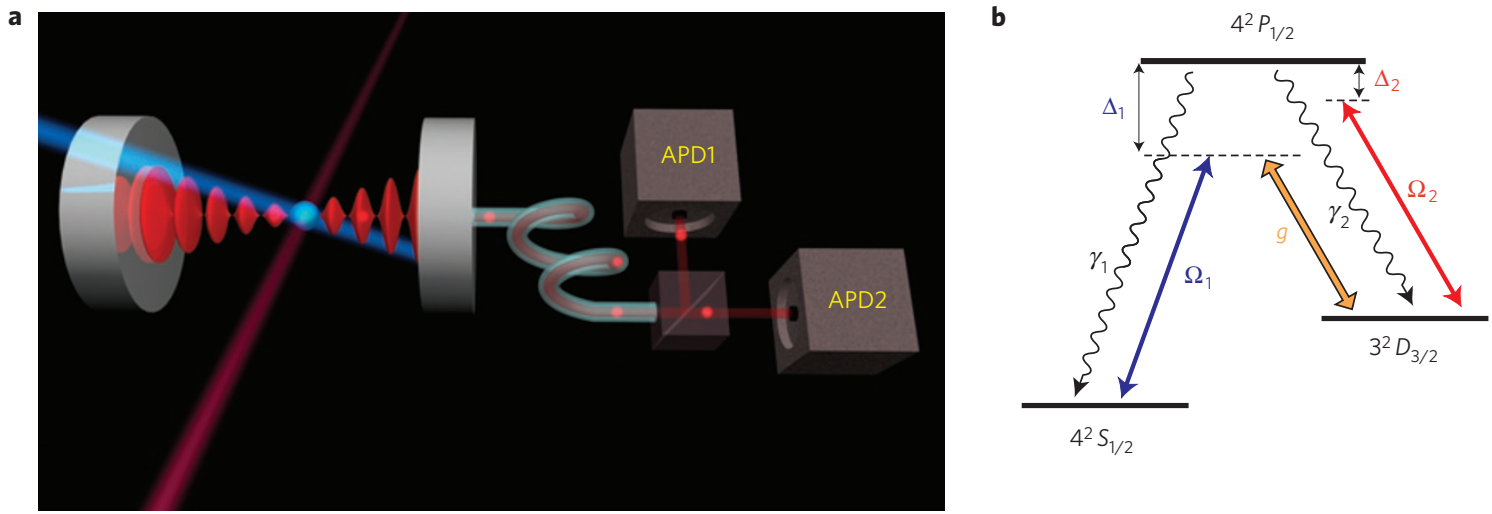

Figure 1 | Experimental set-up and excitation scheme. a, Schematic experimental set-up. The ion (blue ball) is surrounded by a high-finesse cavity and positioned in an anti-node of the standing-wave cavity field. The single-ion device is excited to a meta-stable state through a Raman transition (blue drive-laser beam and cavity). A red laser provides repumping to close the excitation cycle. Photons leaving the cavity are coupled into a multimode optical fibre, split by a non-polarizing beam splitter and detected by two avalanche photodiodes (APD) in a Hanbury-Brown and Twiss set-up. Photon arrival times are recorded with $\approx 300$ ps temporal resolution. $\mathbf{b}$, Relevant level scheme of the ${ }^{40} \mathrm{Ca}+$ ion. A quantization axis is defined by applying a weak magnetic field, $B=2.8 \mathrm{G}$, perpendicular to the cavity axis. The drive laser $\left(\Omega_{1}\right)$ is polarized parallel to the $\mathbf{B}$ field: it excites transitions conserving angular momentum $(\Delta m=0)$. The cavity $(g)$ supports two linear polarizations, perpendicular and parallel to the $\mathbf{B}$ field. The recycling laser $\left(\Omega_{2}\right)$ is polarized perpendicular to the $\mathbf{B}$ field.

this laser also provides weak Doppler cooling to reduce the motion of the ion in the trap ${ }^{22}$.

We carried out numerical simulations using a master-equation approach to predict measured observables, that is, the intra-cavity mean photon number, $n_{\mathrm{ss}}$, the Mandel $Q$ parameter ${ }^{2}$ and the ratio $R$ between coherent and incoherent emission rates on the lasing transition for continuous application of all lasers. The $Q$ parameter, $Q=n_{\text {ss }}\left(g^{(2)}(0)-1\right)$, quantifies intensity fluctuations in the quantum $(Q<0)$ and classical $(Q \geq 0)$ domains. The $R$ parameter is defined as $R=\kappa \cdot n_{\mathrm{ss}} /\left(\gamma_{2} \cdot n_{P}\right)$, where $n_{P}$ is the steady-state population of the $P_{1 / 2}$ level, which controls the amplitude of incoherent photon scattering. Experimentally, $R$ is accessed by monitoring the fluorescence rate on the $S_{1 / 2} \leftrightarrow P_{1 / 2}$ transition, which leads to $n_{\mathrm{P}}$.

In the following, we show that the ion-cavity device acts as a thresholdless laser when operated at the onset of the strongcoupling regime, that is, for $g_{\text {eff }} \sim \gamma_{\text {eff }}^{(1)}$. Raman resonance between selected $S_{1 / 2}$ and $D_{3 / 2}$ states is established at low recycling rates by adjusting the drive-laser detuning ${ }^{22}$. In this situation, the strength of external pumping is given by the recycling-laser parameter, $s_{2}$, which is held at a fixed detuning $\Delta_{2} /(2 \pi)=-20 \mathrm{MHz}$.

Figure 2 shows the measured steady-state photon number $n_{\mathrm{ss}}$ and the $Q$ and $R$ parameters as a function of external pumping together with simulation results (see Supplementary Information) for continuous laser excitation of the trapped ion at the boundary of the strong-coupling regime $\left(g_{\text {eff }} \sim \gamma_{\text {eff }}^{(1)}\right)$. The following three regions are identified. At low recycling parameters (region (I) of Fig. 2), we observe the signature of a thresholdless single-atom laser. It is marked by an increase of the intra-cavity mean photon number, $n_{\mathrm{ss}}$, whereas intensity fluctuations, as indicated by the $Q$ parameter, are reduced. Lasing occurs in the quantum domain $\left(Q=-2.3(4) \%\right.$ for $\left.\Omega_{2} /(2 \pi) \approx 4.6 \mathrm{MHz}\right)$ because single photons are stimulated into the cavity mode through the vacuum field whereas spontaneous emission is reduced $(R \approx 0.7 \cdots 0.8)$. In region (II) of Fig. 2, the laser starts losing its quantum properties as it approaches the classical domain $(Q \geq 0)$, whereas the intra-cavity mean photon number reaches a maximum. For even stronger pumping, the recycling laser introduces phase noise, leading to an increase of the $Q$ parameter (region (III) of Fig. 2). At the same time, it imposes a Stark shift of the $D_{3 / 2}$ levels, effectively reducing the atom-cavity coupling and therefore the mean photon number. This is a manifestation of the theoretically predicted self-quenching of single-atom lasers ${ }^{9,12}$.
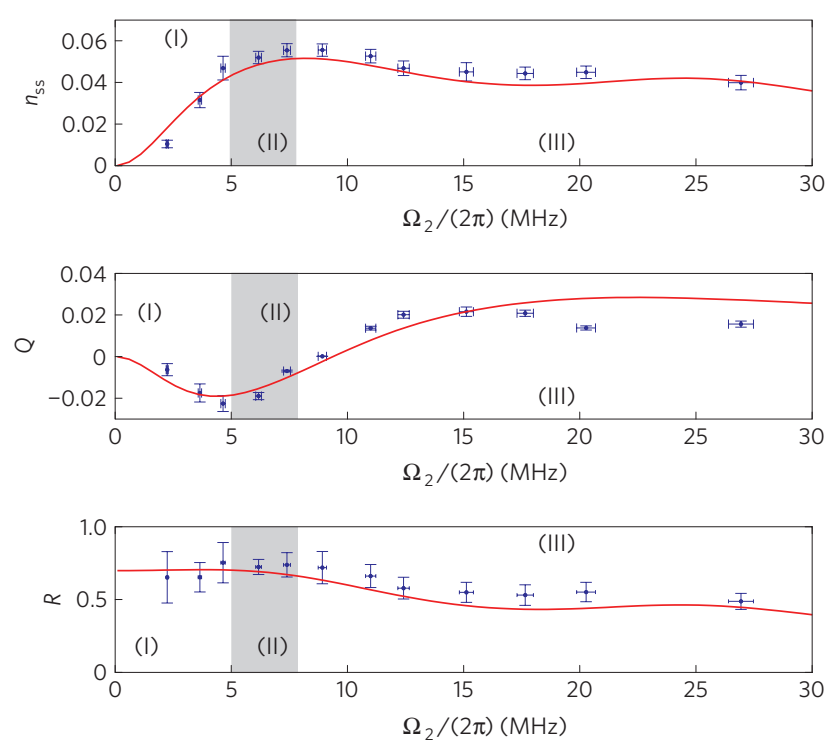

Figure 2 | Thresholdless single-atom laser. Statistical behaviour of the single-ion device at the boundary of the strong-coupling regime. Shown are the mean intra-cavity photon number, $n_{\mathrm{ss}}$, the Mandel $Q$ parameter and the ratio $R$ between coherent and incoherent scattering. Region (I) shows thresholdless lasing of the single-ion device, marked by decreasing intensity fluctuations $(\sim Q)$ and increasing photon flux $\left(\sim n_{\mathrm{SS}}\right)$ as a function of external pumping, whereas $R$ is of the order of one. In regions (II) and (III), the laser turns off (self-quenching). The drive-laser parameters are $\left(\Omega_{1}, \Delta_{1}\right)=2 \pi \cdot(88,-350) \mathrm{MHz}$, leading to $g_{\text {eff }} /(2 \pi)=165 \mathrm{kHz}$ $\sim \gamma_{\text {eff }}^{(1)} /(2 \pi)=170 \mathrm{kHz}$. The recycling rate is varied by the Rabi frequency, $\Omega_{2}$, for a constant detuning $\Delta_{2} / 2 \pi \approx-20 \mathrm{MHz}$. Experimental data are obtained with the same trapped ion continuously probed over $12 \mathrm{~h}$. The solid lines show the results of numerical simulations using independently calibrated experimental parameters. The error bars are derived from calibration uncertainties of the corresponding quantities (see Supplementary Information).

In Fig. 3a-c, we have investigated the pronounced change in the statistics of the emitted light observed in regions (I)-(III) of Fig. 2 in more detail for a slightly different (albeit compatible) set of experimental parameters. Figure 3 a shows the measured and 

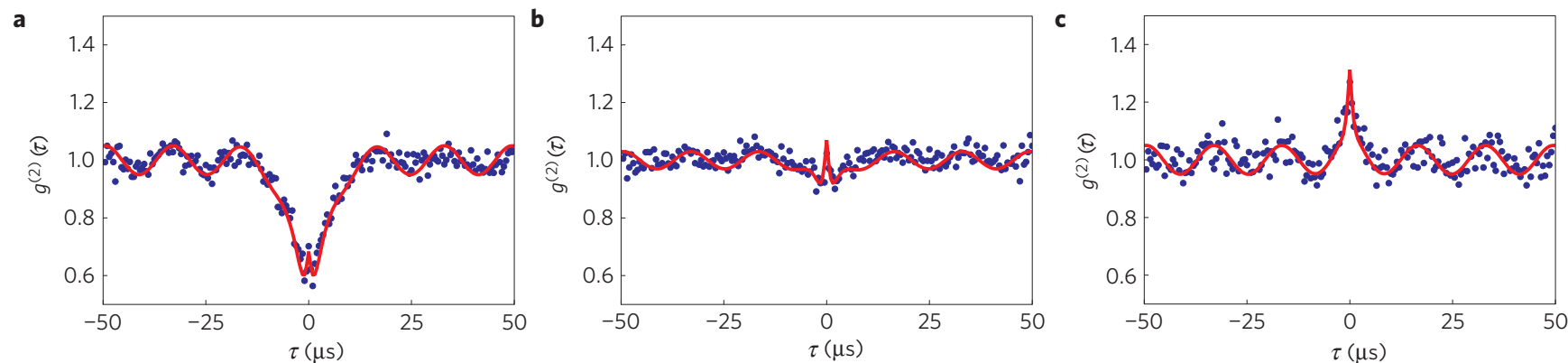

Figure 3 | Tunable photon statistics. a-c, Normalized second-order photon correlations, $g^{(2)}(\tau)$, for different photon generation rates. Increasing the photon generation rate leads to a transition from sub-Poissonian (a) through almost Poissonian (b) to super-Poissonian, bunched (c) light emitted by the cavity. The peak observed around $\tau=0 \mu \mathrm{s}$ is a feature of the intermediate-coupling regime and stems from the accumulation of photons inside the cavity. The drive-laser excitation is such that $\left(\Omega_{1}, \Delta_{1}\right) \approx 2 \pi(95,-400) \mathrm{MHz}$ and the recycling laser is set at $\left(\Omega_{2}, \Delta_{2}\right) \approx 2 \pi(7,-20) \mathrm{MHz}$ in $\mathbf{a}$, at $\left(\Omega_{2}, \Delta_{2}\right) \approx 2 \pi(12,-20) \mathrm{MHz}$ in $\mathbf{b}$ and at $\left(\Omega_{2}, \Delta_{2}\right) \approx 2 \pi(16,-20) \mathrm{MHz}$ in $\mathbf{c}$. In every measurement, the cavity detuning is $\Delta_{\mathrm{c}} / 2 \pi \approx-400 \mathrm{MHz}$, the time resolution is set at $500 \mathrm{~ns}$ and accidental correlations have been subtracted (see Supplementary Information). The solid lines show the results of numerical simulations using independently calibrated experimental parameters.
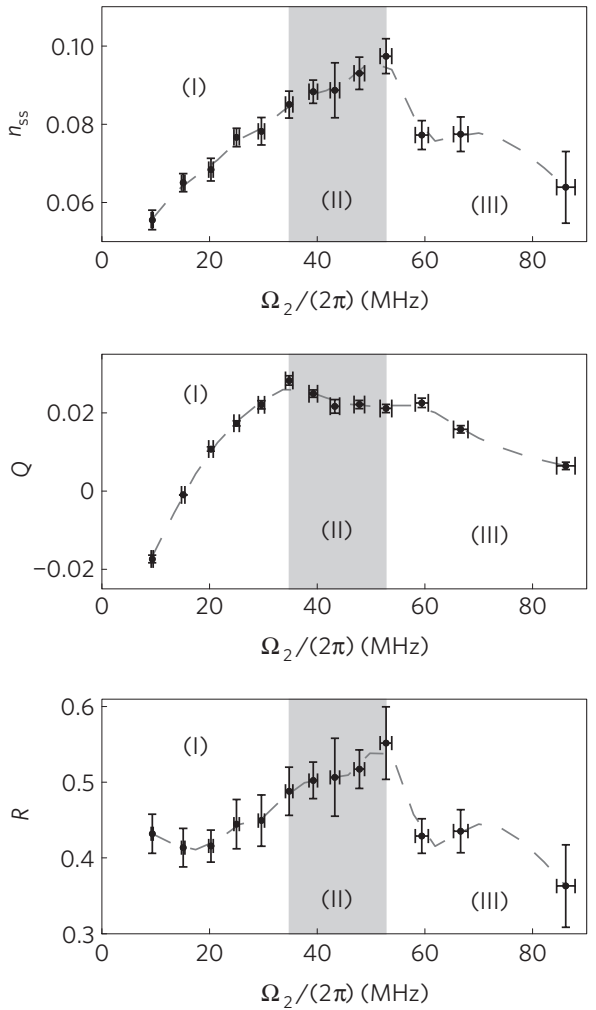
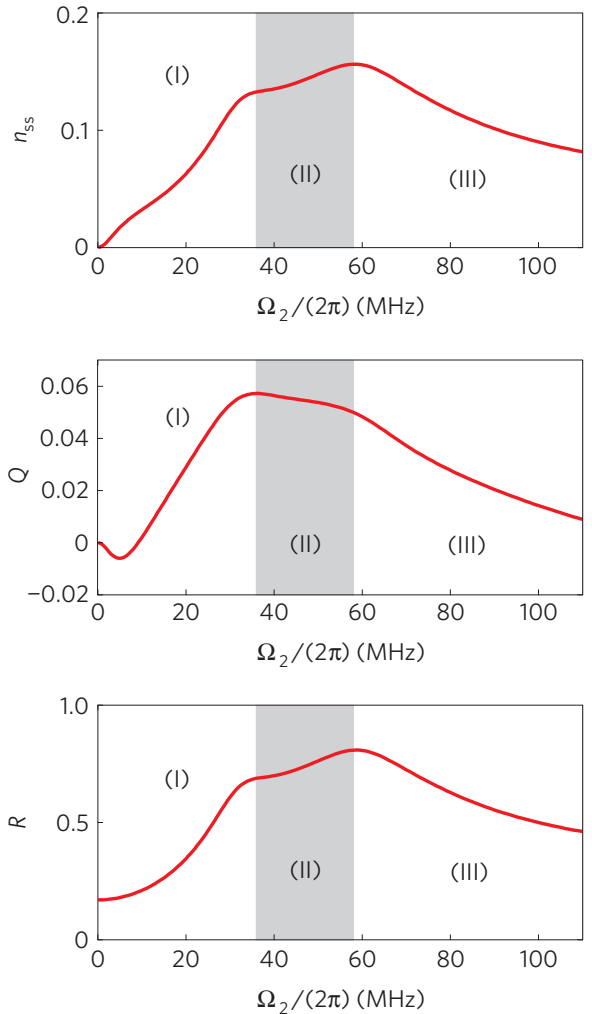

Figure 4 | Single-atom laser with threshold behaviour. a,b, Comparison between experimental observations (a) and theoretical predictions (b) for the statistical response of the single-ion device operating as a laser with threshold behaviour. Shown are the mean intra-cavity photon number, $n_{\text {ss, }}$ the Mandel $Q$ parameter and the ratio $R$ between coherent and incoherent scattering. Intensity $\left(\sim n_{\mathrm{ss}}\right)$ and intensity fluctuations $(\sim Q)$ rise as a function of external pumping in region (I), indicating classical behaviour. Signatures of a laser at threshold are observed in region (II) where $Q$ has passed through a maximum whereas $n_{\mathrm{sS}}$ is still rising and $R$ is of the order of one. Self-quenching turns the laser off in region (III). The drive-laser parameters are $\left(\Omega_{1}, \Delta_{1}\right)=2 \pi(130,-350) \mathrm{MHz}$, resulting in a $g_{\text {eff }} \sim 240 \mathrm{kHz}$. The recycling laser has a fixed detuning $\Delta_{2} / 2 \pi \approx-20 \mathrm{MHz}$. Experimental data are obtained with the same trapped ion continuously probed over $12 \mathrm{~h}$. The dashed lines are a guide to the eye. The error bars are derived from calibration uncertainties of the corresponding quantities (see Supplementary Information).

calculated normalized correlation functions $g^{(2)}(\tau)$ for a small total cycling rate $\Gamma_{\text {tot }} / 2 \pi \sim 100 \mathrm{kHz}$. The slow recycling process introduces a time gap between successive photon emissions and sub-Poissonian light $(Q \sim-1.2(1) \%)$ is emitted ${ }^{11,23}$. Different timescales are apparent in Fig. 3a. The decrease of the correlation function for $|\tau|<1.5 \mu$ s reveals the finite storage time for cavity photons. For longer times $\tau$, the $g^{(2)}$ function approaches unity on a timescale given by the total cycling rate $\Gamma_{\text {tot }}$. Finally, for long time intervals $\tau$, a modulation of the correlation function is observed, which can be attributed to the beat note between the ion's two quasi-degenerate radial modes of motion in the trap ${ }^{24}$. As we slightly increase the recycling parameter, the photon flux remains almost constant, but the statistical properties of the emitted photons change markedly: the correlation function becomes almost flat $(Q=0.0(1) \%$ in Fig. 3b), akin to a Poissonian source of light. At even higher recycling parameters, the output statistics resembles 
that of a thermal source $(Q=+1.0(1) \%$ in Fig. 3c). In general, the measured coincident photon correlations, $g^{(2)}(0)$, are governed by the population of the cavity Fock states with two photons, $p_{2}$, so that $g^{(2)}(0) \sim 2 p_{2} / p_{1}^{2}$, where $p_{1}$ is the population of states with only one photon. The population $p_{2}$ is established through an interplay between the rate $\Gamma_{\text {tot }}$ at which photons are added to the cavity and the rate $2 \kappa$ at which the photon state leaves the cavity (see Supplementary Table S2). The tunability of this population $p_{2}$ and thus the statistical properties of the emitted light is a feature of our system, which can be adjusted to operate in the strong- or weak-coupling regimes, as in the following where we demonstrate a single-atom laser showing threshold behaviour.

In an experiment with different parameters, we studied the single-ion device for an even larger number of photons in the cavity. This was achieved by increasing the drive-laser intensity to $\Omega_{1}=2 \pi \cdot 130 \mathrm{MHz}$. Experimental results are shown in Fig. 4 , where we identify the following regimes. For low recycling rates, that is, in region (I), the photon number and $Q$ parameter both rise, indicating classical non-lasing behaviour. Increasing the external pumping further (region (II)), $n_{\mathrm{ss}}$ still grows whereas the $Q$ parameter has passed through a maximum and decreases. Indeed, we note a $17 \%$ increase of the intra-cavity photon number together with a reduction of 33\% in intensity fluctuations. At the same time, the rate of coherent versus incoherent emission on the lasing transition indicated by the ratio $R$ is of the order of unity. These are the signatures for the onset of a laser threshold ${ }^{11,12}$. As for a conventional laser, the threshold is reached when the population of photons in the cavity exceeds a critical value for which photon-stimulated emission compensates incoherent processes. Most notably, this critical value is less than one for our one-atom device. Furthermore, we note that the variation of $n_{\mathrm{ss}}$ at threshold is less pronounced than for classical lasers. This is a consequence of the low photon number in the lasing mode, as theoretically predicted $^{9,11,12,16,17}$, caused by a saturation of the rate of external pumping $\left(\Gamma_{\text {tot }}\right)$ in region (II). Furthermore, we observe in region (III) quenching of the laser output. Unlike the experiments shown in Fig. 2, this is not due to a level Stark shift because the Raman detuning is chosen such that resonant coupling is established at threshold. Rather, numerical simulations indicate that it is due to the formation of dark states by coherent coupling by means of $\Omega_{2}$, which disables the recycling process. More precisely, the induced dressed states, generally composed of two $D_{3 / 2}$ magnetic sub-states and one $P_{1 / 2}$ sub-level, lose their $P_{1 / 2}$ contribution in region (III), where $\Omega_{2}$ is large. This renders the external pumping inefficient, hence yielding self-quenching, which is a striking difference compared with conventional lasers. In fact, it constitutes an intrinsic limitation of single-atom lasers. In Fig. 4, we also present theoretical simulations, which agree qualitatively with the experimental data. We attribute the discrepancy to the non-zero temperature of the ion, which modifies the excitation spectrum for the parameter range of the experiments shown in Fig. 4 and is not included in the simulations (see Supplementary Information).

We have demonstrated that a laser threshold can be achieved at the single-atom level, although much less pronounced compared with classical lasers ${ }^{9,11,12,16,17}$ as a consequence of the low photon number in the lasing mode. Another striking difference between one-atom and classical lasers is the ability to tune the photon emission statistics from quantum to classical. Such a light source offers perspectives to study new types of spectroscopy, based on narrow-band optical excitation with quantum/classical statistical distribution $\mathrm{s}^{25}$. Our trapped-ion laser is ideally suited to further investigate the transition between quantum and classical lasers through controlled addition of more and more ions interacting with the light field.
Received 12 January 2010; accepted 17 February 2010; published online 28 March 2010

\section{References}

1. Schawlow, A. L. \& Townes, C. H. Infrared and optical masers. Phys. Rev. 112, 1940-1949 (1958)

2. Mandel, L. \& Wolf, E. Optical Coherence and Quantum Optics (Cambridge Univ. Press, 1995).

3. Gardiner, C. \& Zoller, P. Quantum Noise (Springer, 2004).

4. Cohen-Tannoudji, C., Dupont-Roc, J. \& Grynberg, G. Atom-Photon Interactions: Basic Processes and Applications (Wiley, 1998).

5. Milonni, P. W. The Quantum Vacuum (Academic, 1994).

6. Berman, P. R. (ed.) Cavity Quantum Electrodynamics (Academic, 1994).

7. An, K., Childs, J. J., Dasari, R. R. \& Feld, M. S. Microlaser: A laser with one atom in an optical resonator. Phys. Rev. Lett. 73, 3375-3378 (1994).

8. McKeever, J., Boca, A., Boozer, A. D., Buck, J. R. \& Kimble, H. J. Experimental realization of a one-atom laser in the regime of strong coupling. Nature 425, 268-271 (2003)

9. Mu, Y. \& Savage, C. M. One-atom lasers. Phys. Rev. A 46, 5944-5954 (1992).

10. Pellizzari, T. \& Ritsch, H. Preparation of stationary Fock states in a one-atom Raman laser. Phys. Rev. Lett. 72, 3973-3976 (1994).

11. Briegel, H.-J., Meyer, G. M. \& Englert, B.-G. Dynamic noise reduction in multilevel lasers: Nonlinear theory and the pump-operator approach. Phys. Rev. A 53, 1143-1159 (1996).

12. Meyer, G. M., Briegel, H.-J. \& Walther, H. Ion-trap laser. Europhys. Lett. 37, 317-322 (1997).

13. Björk, G., Karlsson, A. \& Yamamoto, Y. Definition of a laser threshold. Phys. Rev. A 50, 1675-1680 (1994).

14. Strauf, S. et al. Self-tuned quantum dot gain in photonic crystal lasers. Phys. Rev. Lett. 96, 127404 (2006).

15. Noginov, M. A. et al. Demonstration of a spaser-based nanolaser. Nature 460 , 1110-1112 (2009).

16. Rice, P. R. \& Carmichael, H. J. Photon statistics of a cavity-QED laser: A comment on the laser-phase-transition analogy. Phys. Rev. A 50, 4318-4329 (1994).

17. Jones, B., Ghose, S., Clemens, J. P., Rice, P. R. \& Pedrotti, L. M. Photon statistics of a single atom laser. Phys. Rev. A 60, 3267-3275 (1999).

18. Jin, R. et al. Photon-number correlations near the threshold of microcavity lasers in the weak-coupling regime. Phys. Rev. A 49, 4038-4042 (1994).

19. Wiseman, H. M. Defining the (atom) laser. Phys. Rev. A 56, 2068-2084 (1997).

20. Birnbaum, K. M. et al. Photon blockade in an optical cavity with one trapped atom. Nature 436, 87-90 (2005).

21. Boozer, A. D., Boca, A., Buck, J. R., McKeever, J. \& Kimble, H. J. Comparison of theory and experiment for a one-atom laser in a regime of strong coupling. Phys. Rev. A 70, 023814 (2004).

22. Russo, C. et al. Raman spectroscopy of a single ion coupled to a high-finesse cavity. Appl. Phys. B 95, 205-211 (2009).

23. Briegel, H.-J., Meyer, G. M. \& Englert, B.-G. Pump operator for lasers with multi-level excitation. Europhys. Lett. 33, 515-520 (1996).

24. Rotter, D., Mukherjee, M., Dubin, F. \& Blatt, R. Monitoring a single ion's motion by second-order photon correlation. New J. Phys. 10, 043011 (2008).

25. Drummond, P. D. \& Ficek, Z. Quantum Squeezing (Springer, 2004).

\section{Acknowledgements}

We acknowledge fruitful discussions with T. Salzburger, H. Ritsch, H.-J. Briegel and P. Zoller. We thank E. S. Phillips for assistance during the early stages of the experiment. This work has been partially supported by the Austrian Science Fund (SFB 15), by the European Commission (QUEST network, HPRNCT-2000-00121, QUBITS network, IST-1999-13021, SCALA Integrated Project, Contract No. 015714) and by the 'Institut für Quanteninformation GmbH'. C.R. acknowledges support from the Fundação para a Ciência e a Tecnologia -SFRH/BD/6208/2001 and A.S. acknowledges support from the Studienstiftung des deutschen Volkes. P.O.S. acknowledges support through the Centre for Quantum Engineering and Space Time Research (QUEST) at the PTB in Braunschweig and University of Hannover.

\section{Author contributions}

F.D. and C.R. contributed equally to this work. All authors contributed to all aspects of this work.

\section{Additional information}

The authors declare no competing financial interests. Supplementary information accompanies this paper on www.nature.com/naturephysics. Reprints and permissions information is available online at http://npg.nature.com/reprintsandpermissions. Correspondence and requests for materials should be addressed to P.O.S. 